RUNNING HEAD: attentional disengagement predicts depression via brooding

\title{
Attentional disengagement from emotional information predicts future depression via changes in ruminative brooding: A five-month longitudinal eye-tracking study
}

\author{
Alvaro Sanchez-Lopez ${ }^{1,2}$, Ernst H. W. Koster ${ }^{2}$, Jill Van Put ${ }^{2}$, and Rudi De Raedt ${ }^{2}$ \\ ${ }^{1}$ Complutense University of Madrid, Spain \\ ${ }^{2}$ Ghent University, Belgium
}

\author{
* Corresponding author: \\ Alvaro Sanchez-Lopez \\ Complutense University of Madrid \\ Department of Personality, Evaluation and Psychological Treatment \\ Faculty of Psychology, Somosaguas Campus \\ 28223, Madrid (Spain) \\ E-mail: alvsanch@ucm.es
}




\begin{abstract}
Brooding is considered a maladaptive form of emotion regulation linking adverse events to increases in depressive symptoms. The "Impaired Disengagement Hypothesis" (Koster, De Lissnyder, Derakshan \& De Raedt, 2011) proposes that attentional disengagement processes are a main mechanism involved in the emergence and maintenance of brooding responses. In this study we tested prospective predictions derived from this framework, relying on eye-tracking to assess direct processes of attentional disengagement from emotional faces (i.e., time to move gaze away from either positive or negative faces when prompted to fixate a different face). A sample of undergraduates $(\mathrm{n}=89)$ completed measures of depression, brooding, and the attentional disengagement task at baseline (beginning of the semester) and five months later (immediately after a stressful period: examination). The results supported a moderated mediation model where slower disengagement from positive faces at baseline (predictor) predicted decreases in brooding during the follow-up period (mediator), indirectly predicting decreased depressive symptoms at follow-up (outcome) in individuals encountering more adverse events during the follow-up period (moderator). Furthermore, analyses also supported a moderation model where more habitual brooding at baseline (predictor) predicted slower disengagement from negative faces at follow-up (outcome) in individuals encountering more adverse events (moderator). Our findings support bidirectional influences between attentional disengagement and brooding and highlight protective attention patterns with implications for the development of efficient strategies for the prevention of depression.
\end{abstract}

Key words: Emotion processing; attentional disengagement; rumination; depression; longitudinal research; eye-tracking. 


\section{Introduction}

Dysfunctional mood regulation in response to adverse or stressful events is a key factor involved in the onset and maintenance of depression (Joormann \& Quinn, 2014). Adverse lifeevents are known to initiate self-focused thoughts on the causes, meanings, and consequences of them and their associated negative moods. This initial response may reflect, in essence, a normative process to initially cope with negative events or low mood. However, when it becomes habitual and exaggerated, such response style, defined as rumination (Susan Nolen-Hoeksema, 1991), may turn into a maladaptive form of mood regulation and cause a number of detrimental effects (Watkins, 2008). Specifically, an habitual ruminative response style characterized by brooding -passively and repetitively focusing on symptoms and consequences of distress (Treynor, Gonzalez, \& Nolen-Hoeksema, 2003)- is considered a key vulnerability factor that in interaction with stressful events leads to depression (e.g., see Lyubomirsky \& Tkach, 2008). Supporting this, frequent use of ruminative brooding is not only a characteristic of currently depressed individuals (Aldao, Nolen-Hoeksema, \& Schweizer, 2010; Joormann, Dkane, \&

Gotlib, 2006; Treynor et al., 2003), but it also predicts future depression onset (Nolen-Hoeksema, 2000). Individuals using more ruminative responses to the occurrence of adverse events have been found to experience subsequent increases in depressive symptoms (Nolen-Hoeksema, McBride, \& Larson, 1997; Nolen-Hoeksema \& Morrow, 1991; Nolen-Hoeksema, Parker, \& Larson, 1994). Yet, although the negative consequences of brooding in response to adverse events have been abundantly demonstrated, it is less clear why some individuals are more prone to experience this type of response to stress.

\section{The impaired disengagement hypothesis}

Current frameworks highlight the importance of dysfunctional attentional processes, including reduced attentional control and attention biases, as potential mechanisms involved in 
the persistence of maladaptive ruminative responses to stress (e.g., Joormann \& Quinn, 2014). This proposal is formulated in detail by the Impaired Disengagement Hypothesis (Koster, De Lissnyder, Derakshan, \& De Raedt, 2011). This model postulates that the existence of difficulties to disengage attention from negative information is a central mechanism putting individuals at risk for prolonged levels of maladaptive rumination and brooding in particular. When confronted with adverse or stressful events, an initial analytic response focused on the negative causes/consequences of the stressors is a normal reaction that may help individuals to regulate their behavior. However, this response style is typically terminated when the problem is solved or, in the absence of an immediate solution, when the individual starts engaging processes to down-regulate the initial negative thoughts and negative mood. In most individuals, this involves disengaging attention from negative information and redirecting their attentional focus towards more positive/adaptive external features (i.e., attention disengagement) and/or internal thoughts (i.e., attentional control), allowing them to reappraise the situation or distract from negative aspects. In contrast, having difficulties to disengage attention from negative external/internal information (for a detailed taxonomy on external and internal attentional functions, see, for instance, Chun, Golomb, \& Turk-Browne, 2010) is viewed as a vulnerability factor. Such disengagement impairments are thought to lead to an increased use of brooding when coping with adverse events, which in turn elicits heightened depressive symptoms: dysfunctional attentional disengagement $\rightarrow$ higher brooding in response to adverse events $\rightarrow$ depressive symptoms (Hypothesis 1). Moreover, the Impaired Disengagement Hypothesis further posits that there are reciprocal relations between brooding and impaired attentional disengagement functions (i.e., reinforcing cycles between brooding and disengagement impairments). Specifically, the habit to use brooding is proposed to lead to impaired reallocation of attention when coping with adverse 
events: habitual brooding $\rightarrow$ dysfunctional attentional disengagement in response to adverse events (Hypothesis 2).

The proposed framework provides clear predictions that may help to identify key temporal pathways of vulnerability to depression, in order to develop preventive interventions. Previous research has already reported evidence on the bidirectional influence between internal attentional control for emotional information and rumination and how they interplay to contribute to future depression. For instance, Zetsche and Joormann (2011) showed that difficulties to inhibit the processing of negative information (negative words and angry faces) from working memory at baseline were predictive of rumination changes after six months. Similarly, De Lissnyder et al. (2012) showed that impaired attentional control over emotional material at baseline interacts with future stress to predict rumination changes at follow-up, more specifically, increased brooding in response to stress. Moreover, Demeyer, De Lissnyder, Koster, \& De Raedt (2012) showed a significant influence of impaired attentional control for angry faces at baseline on depressive symptoms one year later, which was fully mediated by rumination. Finally, there is some preliminary evidence supporting the second hypothesis of the impaired disengagement framework (namely, that habitual rumination would also predict future attentional impairments as a function of experienced stress). For instance, Connolly et al. (2014) showed that adolescents with higher levels of baseline rumination displayed impaired selective attention and attentional switching at a 15-months follow-up.

Overall, this initial set of studies indicates that the temporal interplays between attentional control functions and ruminative tendencies may a) be bidirectional, and b) facilitate depressive symptoms at long time intervals. Yet, previous longitudinal research has focused on testing prospective predictions at the level of disengagement from internal mental representations (i.e., attentional control functions). Similarly, most of these studies focused on testing attentional 
control interplays with global indicators of rumination, rather than focusing on specific maladaptive forms of rumination, namely brooding. Prospective studies in the context of how direct external attention disengagement may interplay with maladaptive ruminative styles (i.e., brooding) to account for depression are still lacking. Nonetheless, previous research provided some initial support for the existence of associations between attentional disengagement and depression, and for a potential mediating role of brooding in accounting for the association between impaired external disengagement and depressive symptoms. Below we discuss the available research to date in these areas.

\section{External attentional disengagement and depression}

Regarding the association between external attention processes and depression, some previous research suggests that attention biases in the processing of negative information influences mood regulation and may contribute to the onset and maintenance of depression. Beevers and Carver (2003) showed that attention biases towards negative material, as measured by reaction times during long 1,000-ms stimuli presentations in a dot-probe task, in interaction with the occurrence of adverse negative events, predicted increases in undergraduates' depressive symptoms seven weeks later. Beevers, Lee, Wells, Ellis and Telch (2011) extended these findings using an eye-tracking task comprising monitoring participants' gaze during free-viewing of emotional stimuli. This study showed that longer total fixation times and longer mean gaze durations on sad faces at baseline assessment were predictive of subsequent higher depressive symptoms as a function of the level of stress exposure of participants. Yet, these previous studies did not measure attention biases in a manner that allows making straightforward conclusions about the specific role of attentional disengagement in depression.

The most direct evidence of the role of attentional disengagement impairments in depression derives from studies using a novel eye-tracking task, namely the engagement- 
disengagement task (Sanchez, Romero, \& De Raedt, 2017; Sanchez, Vazquez, Marker, LeMoult, \& Joormann, 2013). In this task, participants freely watch pairs of emotional (e.g., happy, sad, or disgusted) vs. neutral faces for $3,000 \mathrm{~ms}$, and then the stimuli presentation does not continue until the eye-tracker detects a new fixation on the emotional face. When this occurs, a frame (i.e., a square or a circle) appears surrounding the neutral face, and participants are instructed to reallocate their attention to the frame in order to indicate its shape as quickly as possible. The time to move gaze from the emotional towards the neutral face is recorded. Using this task, it has been shown that, compared to healthy controls, both clinically (Sanchez et al., 2013) and subclinically depressed participants (Sanchez et al., 2017) take longer to move their gaze away from negative (sad, disgusted) faces towards neutral counterparts. Importantly, such difficulties in attentional disengagement predicted impaired mood recovery after confronting transient lab-stressors (i.e., anticipating and giving a speech), with participants characterized by such disengagement impairments showing maintained negative mood states after confronting the stressors (Sanchez et al., 2013, 2017).

\section{External attentional disengagement and brooding}

The impaired disengagement framework proposes that the role of such impairments in external attentional disengagement to predict sustained negative affect and future depression would specifically occur through the influence of impaired disengagement on the use of maladaptive forms of emotion regulation (i.e., brooding). Despite the absence of direct tests of these predictions, several cross-sectional studies support a covariation between depression-related attention biases and brooding rumination. Studies using the dot-probe task have demonstrated an association between the use of habitual rumination (Donaldson, Lam, \& Mathews, 2007) and particularly brooding (Joormann et al., 2006; Southworth, Grafton, MacLeod, \& Watkins, 2017) with larger attention biases for negative words at 1,000-ms stimuli presentations. Similarly, eye- 
tracking studies using free-viewing paradigms have shown that habitual brooders are characterized by both more sustained attention to sad faces and less sustained attention to happy faces (Duque, Sanchez, \& Vazquez, 2014; Owens \& Gibb, 2017), a double bias that is commonly observed in depressed individuals (see Armstrong \& Olatunji, 2012). Such associations in these studies were independent of concurrent levels of depression, suggesting that they may reflect factors of vulnerability rather than consequences of current depression states.

The most direct evidence for an interplay between attentional disengagement and rumination to prospectively account for affective symptoms comes from a recent study of Valenas, Szentagotai-Tatar, Grafton, Notebaert, Miu, \& Macleod (2017). Using a reaction timebased dot-probe paradigm, these authors have shown that higher levels of baseline rumination predict more difficulties in attentional disengagement from negative information during a stressful pre-exam period 2 weeks later, with higher attentional impairments mediating the influence of baseline rumination on subsequent pre-exam state anxiety at the follow-up. Nonetheless, this study only tested a pathway comprising the role of habitual rumination in predicting future attentional disengagement impairments with the occurrence of stress (i.e., Impaired Disengagement framework's Hypothesis 2). Thus, it remains unknown whether an inverse pathway would also be supported (i.e., attentional disengagement impairments prospectively predict changes in maladaptive ruminative responses at the occurrence of stress, in last term accounting for depression levels; Impaired Disengagement framework's Hypothesis 1). Second, different to most cross-sectional studies, supporting a specific association between attentional disengagement and ruminative brooding, Valenas et al. (2017) relied on a more global assessment of rumination. Therefore, there is a lack of systematic examination of bidirectional temporal interplays between the processes (i.e. attentional disengagement impairments and specific brooding responses) thought to be most centrally implicated in depression risk (Treynor 
et al., 2003). Third, the scarce longitudinal research in this area (Valenas et al., 2017) has studied attention-rumination relations over a short-term period (i.e., 2 weeks) to account for their immediate effects on the occurrence of stress (i.e., state anxiety). In contrast, predictions from the Impaired Disengagement framework take into account the enduring effects of these cognitive factors in accounting for depression onset and maintenance at the long term. Related research on internal attentional control functions has consistently explored bidirectional interplays and supported pathways accounting for depressive symptoms at extended follow-up periods (e.g., Zetsche \& Joormann, 2011: 6 months; Demeyer et al., 2012: 12 months). Finally, previous research on prospective associations between attention and rumination (Valenas et al., 2017) relied on a proxy estimation of attention disengagement (i.e., derived from reaction times in a dot-probe task), which has several inherent drawbacks. First, reaction time-based proxy estimations entail a distal relation between the behavioral output (i.e., key presses) and the examined attentional processes. Second, there are currently many concerns on the poor psychometric properties of these assessments (e.g., Waechter, Nelson, Wright, Hyatt, \& Oakman, 2014). Overall, these issues highlight the necessity of using fine-grained eye-tracking techniques to test the hypotheses derived from the Impaired Disengagement framework in a straightforward way.

In sum, direct tests of bidirectional prospective relations between specific processes of external attentional disengagement (i.e., gaze behaviors) with specific indicators of brooding and, ultimately, the role of such interplays to prospectively account for future depression risk at the long-term are still lacking.

\section{The present study}

Our study aimed to fill this gap in literature by relying on longitudinal assessment of depressive symptoms and ruminative brooding as well as attentional disengagement processes, as 
directly indexed with the eye-tracking engagement-disengagement task (Sanchez et al., 2013, 2017). Importantly, the engagement-disengagement task provides reliable and direct indices of gaze-disengagement from emotional information (i.e., negative and positive facial expressions) when prompted to engage with neutral information (i.e., a neutral expressions of the same actor) at late-stage processing (after 3,000 ms of freely watching the face pair; Sanchez, Vanderhasselt, Baeken, \& De Raedt, 2016; Sanchez et al., 2017). We recruited an unselected sample of undergraduate students that were assessed with respect to attentional disengagement biases, brooding, and depressive symptoms at the beginning of the academic course. A second assessment of these indices was performed five months later, using a follow-up period similar to previous studies testing the relationship among attentional inhibition of negative information, ruminative responses, and depression (Zetsche \& Joormann, 2011). Brooding was assessed in relation to its occurrence during the entire follow-up period (i.e., during the past five months). Depression was assessed in relation to its occurrence at the moment of the follow-up (i.e., during the past two weeks), which took part immediately after the mid-term exam period (a period with prominent naturally occurring stressful events in this population). This assessment also included self-reports on the frequency of adverse events experienced during the follow-up period, which was used to test the moderating role of stress frequency on prospective interplays between attentional disengagement and brooding. Overall, our design allowed us: a) to model bidirectional influences between attentional disengagement and brooding changes, and b) to analyze the contribution of these models to account for changes in depressive symptoms, as a function of the frequency of encountering adverse events.

The following specific hypotheses were tested. First, based on previous studies showing an association between brooding and both longer times fixating on negative information and shorter times fixating on positive information (e.g., Owens \& Gibbs, 2016), we expected that 
both longer times to disengage gaze from negative faces and faster times to disengage gaze from positive faces would prospectively predict changes in brooding, when being confronted with adverse events (cf. Beevers \& Carver, 2003). Specifically, according to the main prediction of the impaired disengagement framework (i.e., Hypothesis 1 of the framework), we hypothesized a moderated mediation model (Figure 1a), where late-stage attentional disengagement biases at the baseline (main predictors) predict brooding changes during the follow-up (mediator) as a function of adverse events' occurrence during that period (moderator), with brooding changes accounting for changes on depressive symptoms at follow-up (outcome). Second, in line with the bidirectional pathway of influence predicted by the impaired disengagement framework (i.e., Hypothesis 2 of the framework: when brooding becomes habitual this also lead to subsequent impairments in attention reallocation in the event of stress experiences; Koster et al. 2011), and with recent research supporting a predictive role of habitual brooding on future attention disengagement biases during stressful periods (Valenas et al., 2017), we hypothesized a moderation model (Figure 1b), where habitual brooding at the baseline (predictor) predicts changes on attention disengagement biases at follow-up (outcome), as a function of adverse events experienced during the follow-up period (moderator).

\section{Methods}

\section{Participants and Procedure}

An unselected sample of 120 undergraduates ( 83 women) of Ghent University, with a mean age of 22.28 years $(\mathrm{SD}=4.44)$ were initially recruited via internet posting to participate in the first session of assessment of the study. The first assessment (T1) was completed at the start of the academic year (September-October 2015). This first session was part of a larger cross-sectional project, where participants completed several questionnaires during the session, including the initial assessment of depressive symptoms level (Beck Depression-Inventory-II, BDI-II; Beck, 
Steer, \& Brown, 1996) and brooding (Brooding subscale of the Ruminative Responses Scale, RRS; Nolen-Hoeksema \& Morrow, 1991), followed by a battery of cognitive tests, including the eyetracking engagement-disengagement task. Participants received 20 euros for their participation in the first assessment.

Five-months after completing the T1 assessment, 112 participants ( 8 participants in $\mathrm{T} 1$ were no longer students at the University at that time or could not be reached) were contacted again to take part in the second assessment of the longitudinal design (T2). This period (February-March 2016) coincided with the end of the first semester examination period. The T2 assessment comprised a questionnaire package, including the second assessment of depressive symptoms (BDI-II) and ruminative brooding (RRS Brooding subscale), together with a questionnaire assessing the number of encountered adverse events during the 5-months follow-up period (Adverse Events Questionnaire, AEQ; Carver, 1998). The T2 assessment also included the completion of the eye-tracking engagement-disengagement task for a second time. In order to reduce drop-outs in the follow-up as much as possible, participants were given the opportunity to only complete the questionnaire package, if they did not have sufficient time to take part in the eyetracking part of T2 assessment. Eighty-nine participants ( 65 women, mean age: 22.45 years, $\mathrm{SD}=$ 4.93) filled out the questionnaires during the T2 assessment (79\% of the contacted sample), with 66 of them (46 women, mean age: 22.92 years, $\mathrm{SD}=5.44$ ) undergoing the full $\mathrm{T} 2$ assessment, also performing the eye-tracking task (59\% of the contacted sample).

All participants were native Dutch speakers and had normal or corrected to normal (using glasses or contact lenses) vision. They provided written informed consent at both sessions. Participants who only completed the second questionnaire package at the 5-months follow-up received 5 additional euros, whereas those who also completed the second eye-tracking assessment 
were compensated with 25 additional euros. The study was approved by the institutional ethical review board at Ghent University.

\section{Self-reported measures}

Depressive symptoms level. The BDI-II (Beck et al., 1996; Dutch translation by Van der Does, 2002) is a 21-item self-report measure of depressive symptom severity. Respondents reported the severity of their experience for each depression symptom on a four-point scale, ranging from 0 to 3 . BDI-II total scores can range from 0 to 63 . This measure has shown excellent reliability and validity (Beck et al., 1996). In the current study, the instrument showed a good internal consistency at both assessments (T1: $\alpha=.85 ; \mathrm{T} 2: \alpha=.86)$.

Brooding. Measures of brooding were derived from the brooding subscale of the RRS (Nolen-Hoeksema \& Morrow, 1991; Dutch translation by Raes \& Hermans, 2007). The RRS is a 22-item questionnaire with a 4-point scale (from 1- "almost never" to 4- "almost always"), that assesses how participants tend to respond to sad feelings and symptoms of dysphoria. The RRS assesses responses to dysphoric moods that are focused on the self (e.g., "You think about all your shortcomings, failings, faults, mistakes"), on symptoms (e.g., "You think about how hard it is to concentrate"), or on possible consequences and causes of moods (e.g., "You analyze recent events to try to understand why you are depressed"). Two subscales are derived from the RRS, the Brooding scale and the Reflection scale (Treynor et al., 2003). The main variable of interest, brooding, is thought to reflect maladaptive forms of ruminative thinking, such as a preoccupation with depressing memories or thoughts. In the current study, there was good internal consistency for the Brooding subscale (T1: $\alpha=.71 ; \mathrm{T} 2: \alpha=.80$ ).

Frequency of adverse events during the 5-months follow-up period. The AEQ (Carver, 1998) was used to measure the frequency of experiencing adverse events during the last five 
months. The AEQ is a 4 items self-report questionnaire especially designed for student populations and is intended to track the occurrence of adverse events commonly occurring in student lives. Two items measure adverse events in the domains of academics and relationships, one item measures adverse events in any other domain, and one item measures the impact of accumulated minor negative events. Participants respond using a scale from 0 to 3 indicating the frequency of adverse events in each domain (i.e., $0=$ No, $1=$ Yes, this happened to me once, $2=$ Yes, this happened to me twice, $3=$ Yes, this happened to me more than twice). Previous research using this questionnaire has demonstrated a) an interaction between experienced stress and impairments in attentional control for negative faces to predict future rumination (De Lissnyder et al., 2012); and b) that the covariation of experienced stress and ruminative responses prospectively predicts future depressive symptom levels (Vanderhasselt, Brose, Koster, \& De Raedt, 2016), which provides the basis for its use in our longitudinal design.

\section{Eye-Tracking Engagement-Disengagement Task}

The engagement-disengagement task (Sanchez et al., 2013) was used to derive direct indices of attentional disengagement from emotional information at both $\mathrm{T} 1$ and $\mathrm{T} 2$. The task comprised 144 randomly presented trials at each of the two assessments. On each trial of this task a pair of pictures of the same person is shown, depicting emotional (i.e., disgusted, happy) and neutral facial expressions. Twenty-four disgusted, happy, and neutral models (12 men and 12 women depicting each of the three emotions) were selected from the Radboud Faces database (RaFD; Langner et al., 2010), based on data from a previous validation (Langner et al., 2010). We relied on the use of disgusted faces, since previous research has shown that such emotional expressions are related to a large degree of social-threat perception compared to angry faces (Amir et al., 2005), and problems in the processing of this kind of information is thought to contribute to both sustained negative affect and post-event rumination (Kashdan \& Roberts, 
2007). Moreover, previous research using the engagement-disengagement task (Sanchez,

Romero, \& De Raedt, 2017) has shown that individuals with higher depression levels are characterized by longer times to disengage gaze from both sad and disgusted faces, and that they are characterized by sustained attention to disgusted over happy facial expressions, with these biases predicting poor stress regulation.

The longitudinal part of the current study employed the basic design of this task (Sanchez et al., 2013), where each trial starts with the presentation of a blank screen for $500 \mathrm{~ms}$, followed by the display of a central fixation cross. Immediately after the participant makes a visual fixation to the area of the fixation cross, a pair of faces (one negative or positive face vs. the neutral face of the same actor) is presented for a pre-specified amount of time (standard time: 3,000 ms). Once the free-viewing time finishes, a "wait for fixation" period is introduced, where stimuli presentation does not continue until participants fixate on a given face (pre-specified in each trial) for $100 \mathrm{~ms}$. Once this occurs, a frame consisting of either a square or a circle appears surrounding the opposite face. Participants are instructed to direct their gaze toward that frame as quickly as possible and press one of two response keys on the keyboard to indicate the type of frame (i.e., square or circle). This task has proven to reliably index patterns of late-stage attentional disengagement from emotional information (i.e., moving gaze away from an initially fixated negative or positive face to a subsequently framed neutral face) in comparison to patterns of attentional engagement (i.e., moving gaze away from an initially fixated neutral face to engage with a subsequently framed negative or positive face; Sanchez et al., 2016, 2017) ${ }^{1}$.

\footnotetext{
${ }^{1}$ As part of a larger project testing several research questions, the full attention task also comprised a set of trials assessing early-stage attention disengagement patterns. Specifically, two "wait for fixation" periods were introduced in the full attention task employed in this project: in half of the trials the "wait for fixation" period was introduced at the beginning of the stimuli presentation, right after the central fixation cross (i.e., at the moment that participants made the first visual fixation in the corresponding target face the frame appeared surrounding the opposite face), whereas the other half comprised the standard late-stage disengagement task, as described above. This was used to index attentional disengagement biases at two separate phases of processing, namely, early and late stages of
} 
Whereas emotional vs. neutral stimulus sets are largely employed in attention research, gaze-disengagement reflects oculomotor behaviors that may be dependent on other additional factors rather than only on competing stimuli valences, such as differences on their arousal levels (e.g., Calvo \& Lang, 2004) or differential perceptual characteristics such as teeth visibility (e.g., Blanco, Serrano-Pedraza, \& Vazquez, 2017). To control for additional confounders, the task in this study also included conditions of competing positive vs. negative faces of the same actor (matched on arousal levels and showing similar perceptual characteristics), allowing also measuring disengagement biases from each emotion when prompted to engage with the opposite emotion (i.e., together with standard conditions of disengagement from each emotion when prompted to engage with a neutral counterpart). In this second disengagement condition participants were prompted to disengage attention from one of the emotions (e.g., negative face) to direct their gaze to the other emotion (e.g., positive face). Therefore, together with standard measures of attentional engagement with emotional information: time to move gaze away from a neutral to a disgusted or happy face (32 trials, 16 of each emotional condition), and standard disengagement from emotional information: time to move gaze away from a disgusted or happy face to a neutral face (32 trials, 16 of each emotional condition), a third condition was included, comprising viewing competing disgusted vs. happy faces (32 trials). This third condition allowed to index: a) the time to disengage attention from negative faces when prompted to engage with positive faces (16 trials), and b) the time to disengage attention from positive faces when

\footnotetext{
emotional processing, and analyze the differential associations between anxiety and depressive symptom levels at each of these stages. These results, available upon request, show that participants with high trait anxiety levels (assessed by the Trait-State Anxiety Inventory; STAI, Spielberger et al., 1970) were characterized by higher disengagement impairments at early compared to late-stages phases of processing. Importantly, such anxiety-related effects at early-stage disengagement were not accounted either by depression or brooding levels. Given this and nonhypothesized prospective relations for early-stage processing biases in the impaired disengagement framework, analyses in the longitudinal study reported here were focused on the study of attentional disengagement biases at the late-stage phase condition of emotional processing, as measured in the trials comprising the basic design of the task, as reported above.
} 
prompted to engage with negative faces (16 trials). A fourth filler condition comprising neutral vs. neutral faces was also included (48 trials), where the neutral expression of the same actor was presented twice and participants had to move their gaze from one to the other exemplar to detect the frame. Corresponding disgusted, happy and neutral expressions in each condition were presented equally often on the left as on the right positions. Both types of frames were also equally likely to appear in the left and right positions in all conditions. The task also included 10 practice trials, followed by a brief pause before starting the actual trials. An overview of the trial sequence and measures of each late-stage disengagement bias index is depicted in Figure 2.

Similar to previous studies (e.g., Sanchez, Vanderhasselt, et al., 2016; Sanchez et al., 2017), criteria for identifying a first shift in gaze to the stimuli surrounded by the frame on each trial were: a) participants were fixated on the target stimulus before the frame on the opposite stimulus appeared, b) eye movements occurred at least $100 \mathrm{~ms}$ after the frame appeared, c) gaze was directed to the stimulus surrounded by a frame rather than remaining at the opposite position or being directed to a different area on the screen (i.e., trials with participants taking longer than 1,000 ms to make a visual fixation on the frame were excluded), and d) participants made a fixation of at least $100 \mathrm{~ms}$ to the stimulus surrounded by a frame. An average of $95 \%$ trials per participants (96\% at T1, 94\% at T2) was identified as valid recordings. From those trials, attentional disengagement bias indices were computed for each of the following conditions: a) time to disengage attention from negative faces (when prompted to engage with neutral faces, and when prompted to engage with positive faces), and b) time to disengage attention from positive faces (when prompted to engage with neutral faces, and when prompted to engage with negative faces). This resulted in a total of four attentional disengagement bias indices at each of the two assessment times. Internal consistencies (Cronbach's alphas) for these indices were all good both 
at T1 and T2 (see Table 1), and comparable to the ones observed for these indices in previous studies (e.g., Sanchez et al., 2017: alphas ranging from .75 to .86).

\section{Data analysis plan}

All analyses were performed using the PROCESS SPSS macro (Hayes, 2012). Hypothesis 1 (moderated mediation models: attentional disengagement $\mathrm{T} 1 \rightarrow$ brooding change $\rightarrow$ depression change, moderated by adverse events) was tested considering each of the four attentional disengagement indices as predictors, using the PROCESS Model 7 (moderated mediation analysis, with the moderator accounting for the relation between the predictor and the mediator). The analytic plan for each of the four moderated mediation models tested comprised three serial steps, in accordance to recommended standard practices for these analyses (Muller, Judd, \& Yzerbyt, 2005; Yzerbyt, Muller, Batailler, \& Judd, 2018): a) test of the interaction effect of the predictor by the moderator on the mediator; b) test of the relationship between the mediator and the outcome partialling out the effects of the predictor and the moderator; and c) bootstrapping estimating the statistical support for a significant conditional indirect effect (i.e., test of the moderated mediation index). Hypothesis 2 (moderation models: brooding $\mathrm{T} 1 \rightarrow$ attentional disengagement change, moderated by adverse events) was tested using the PROCESS Model 1 (moderation analysis), separately considering each of the four attentional disengagement indices as outcomes.

Therefore, in the Moderated Mediation Hypothesis 1: attentional disengagement T1 $\rightarrow$ brooding change $\rightarrow$ depression change, moderated by adverse events $(n=89)$, step $A$ comprised testing how baseline attentional disengagement biases from emotional information at $\mathrm{T} 1$ prospectively predicted changes from $\mathrm{T} 1$ to $\mathrm{T} 2$ in ruminative brooding as a function of adverse events from T1 to T2. Moderation models were tested, one for each of the four attentional disengagement indices at $\mathrm{T} 1$ as predictors, with brooding change as outcome, and 
adverse events' frequency as potential moderator. Brooding change was computed using standardized residuals, allowing controlling for individual differences in brooding at T1 baseline. Brooding scores at $\mathrm{T} 1$ were entered in a simple regression model as predictor of brooding scores at $\mathrm{T} 2$, with the resulting standardized residuals serving as a measure of brooding change. Using standardized residuals is a reliable method to control for variability among individual differences in baseline scores (Segal et al., 2006), and its interpretation is identical to the one of regression models introducing T1 scores of the outcome at T2 in the first step of the model (e.g., Beevers \& Carver, 2003). Our analyses comprised two tests for each of our two predictions (i.e., time to disengage gaze from disgusted faces to engage either with neutral or positive faces positively predicts brooding changes, as a function of adverse events; time to disengage gaze from happy faces to engage with either neutral or disgusted faces negatively predicts brooding changes, as a function of adverse events). Importantly, coefficients were calculated correcting for false discovery rate using the Benjamini-Hochberg method (Benjamini \& Hochberg, 1995) among each of the two tests performed for each hypothesis. When a significant interaction was supported after controlling for the false discovery rate, the interaction effect was followed-up through conditional regressions, examining the predictive role of the given attentional disengagement index on brooding change at high and low levels of adverse events' frequency (1 SD above and below the mean; Aiken \& West, 1991). Step B comprised testing the relation between changes in brooding during follow-up and depressive symptom changes at follow-up, while controlling for the corresponding attentional disengagement predictor at T1 and the moderator. Depressive symptom changes were also computed via standardized residuals (i.e., depression scores at T1 predicts depression scores at $\mathrm{T} 2$, and resulting standardized residuals are saved as a measure of depressive symptom change). When both steps A and B were supported, step C comprised testing the support for the hypothesized moderated mediation models (Fig 1a): i.e., whether those 
attentional disengagement indices at $\mathrm{T} 1$ predicting brooding change, acted as conditional indirect predictors of depressive symptom change at follow-up via brooding change within the follow-up period, as a function of adverse events during that period.

The Moderation Hypothesis 2: brooding $\mathbf{T 1} \rightarrow$ attentional disengagement change, moderated by adverse events $(n=66)$, was used to test whether baseline brooding levels at T1 prospectively predicted changes from $\mathrm{T} 1$ to $\mathrm{T} 2$ in attentional disengagement biases as a function of adverse events experienced during the follow-up period. Indices of attentional disengagement change were computed via standardized residuals and moderation analyses run in PROCESS were controlled for false discovery rate and subsequently decomposed using the same procedures described above.

\section{Results}

\section{Sample characteristics and attrition analyses}

Table 1 summarizes descriptive information of the sample at each assessment time and measure, as well as internal consistencies for each measure. Table 2 summarizes the correlations between each of the variables of interest at each time point.

Completers of the questionnaire package assessment at T2 $(n=89)$ did not differ from non-completers of T2 assessment $(\mathrm{n}=31)$ in their depressive symptom levels, $t(119)=0.29, p=$ .77 , although they showed significantly higher ruminative brooding levels at $\mathrm{T} 1, t(119)=2.25, p=$ .03. Nonetheless, $\mathrm{T} 2$ completers' ruminative brooding levels $(\mathrm{M}=10.97, \mathrm{SD}=3.27$, range: 5 to 20) at $\mathrm{T} 1$ were comparable to the ranges assessed at $\mathrm{T} 1$ for the whole sample $(\mathrm{M}=10.59, \mathrm{SD}=$ 3.24, range: 5 to 20). Furthermore, completers of the eye-tracking assessment at $\mathrm{T} 2(\mathrm{n}=66)$ did not differ from non-completers of the T2 eye-tracking assessment $(n=54)$ in any of the disengagement indices at T1, all $t$ 's $<1.05$, all $p$ 's $>.28$. Analyses showed no gender differences 
in any of the measures of the study either at T1 or T2 assessments, all $t$ 's $<1.51$, all $p$ 's $>.13$.

Therefore, gender was not added as covariate in our longitudinal models ${ }^{2}$.

\section{Moderated Mediation Hypothesis 1 (attentional disengagement $\rightarrow$ brooding $\rightarrow$ depression,}

\section{by adverse events)}

Step A: Attentional disengagement prospectively predicts changes in brooding as a function of adverse events. Results from this step are summarized in Table 3. Attentional disengagement from negative information did not account (either when prompted to engage with neutral or with positive information) for significant changes in brooding, neither directly, nor in interaction with adverse events' frequency. Attentional disengagement from positive to neutral information did not significantly directly predict changes in brooding, but showed a significant interaction with adverse events' frequency. Conditional regressions showed that the index did not predict brooding changes at low levels, $B=2.59, S E=2.20, t=1.18, p=.24$, but, at high levels of adverse events, with longer times to disengage from positive to neutral information predicting decreases in brooding, although this effect was a trend, $B=-4.66, S E=2.48, t=-1.88, p=.06$. Similarly, attentional disengagement from positive to negative information did not significantly directly predict changes in brooding, but showed a significant interaction with adverse events' frequency. Conditional regressions showed that attentional disengagement from positive to negative information did not predict changes in brooding at low levels of adverse events, $B=$

\footnotetext{
${ }^{2}$ We also conducted a 2 (Assessment Time) x 2 (Emotion to Disengage from: Happy, Disgusted) x 2 (Framed Face to Engage with: Neutral, Opposite Emotion) in order to examine significant differences between disengagement biases at each assessment time. Analyses showed a significant Emotion to Disengage from x Framed Face interaction, $F(1,65)=6.74, p=.01, \eta_{p}{ }^{2}=.10$. Bonferroni-corrected post-hoc analyses showed that at both assessment times, when prompted to direct gaze to the framed opposite emotion, participants took longer to disengage from positive to engage with negative faces than to disengage from negative to engage with positive faces, $p=.005$. Similarly, at both assessment times, participants took longer to disengage from positive to engage with negative faces than to disengage from positive to engage with neutral faces, $p=.01$. Therefore, participants in general showed a bias to sustain longer attention in positive information when having to disengage from it, in order to engage with a negative counterpart.
} 
2.07, $S E=2.42, t=0.86, p=.39$, but did negatively predict brooding changes at high levels of adverse events, $B=-5.58, S E=2.15, t=-2.59, p=.01$. The significant effect is illustrated in Figure 3.

Step B: Ruminative brooding change is related to depressive symptom change, controlling for attentional disengagement at T1 and adverse events. The regression model in the second step, considering depression change as outcome, showed that brooding change during the follow-up period was positively related to depressive symptom change at the follow-up: $B=0.25$ ( $\mathrm{SE}=$ $0.11), t=2.31, p=.02$, when partialling out the effects of the predictor (i.e., attentional disengagement from positive to negative at T1) and the moderator. In contrast, the direct effect of attentional disengagement from positive to negative at $\mathrm{T} 1$ on depressive symptom change was not significant: $B=-1.10(\mathrm{SE}=1.84), t=-0.60, p=.55$. Thus, larger decreases in depressive symptom levels from T1 to T2 were related to larger decreases in ruminative brooding during the follow-up period.

Step C: Attentional disengagement indirectly predicts changes in depressive symptoms via changes in ruminative brooding as a function of frequency of adverse events. The final step of Hypothesis 1 comprised the examination of the significance for the moderated mediation index on depressive symptom change from T1 to T2 (outcome), for those attentional disengagement indices at T1 with demonstrated predictive power on brooding changes (i.e., attentional disengagement from positive to negative at T1: predictor), with brooding change as potential mediator and the frequency of adverse events as the moderator. The model was tested using 5000 bootstrap samples and a confidence interval of $95 \%$ (bias-corrected). The moderated mediation index was significant: $-0.86(\mathrm{SE}=.67), 95 \% \mathrm{CI}-2.9169--0.0002$, supporting a conditional indirect effect of attentional disengagement from positive information as predicted: longer times to disengage from positive information at $\mathrm{T} 1$ indirectly predicted depressive symptom decreases 
at follow-up, via their predictive role on brooding decreases during the follow-up period, in those individuals who experienced higher levels of adverse events.

\section{Moderation Hypothesis 2 (brooding $\rightarrow$ attentional disengagement, by adverse events)}

Results from these moderation analyses are summarized in Table 4. Brooding significantly predicted changes in attentional disengagement from negative to neutral information, with this direct effect being accounted by a brooding $\mathrm{x}$ adverse events' frequency interaction. Conditional regressions showed that higher brooding at $\mathrm{T} 1$ predicted faster attentional disengagement from negative to neutral information at low levels of adverse events, $B=-0.13$, $S E=0.05, t=-2.75, p=.01$. In contrast, higher brooding at $\mathrm{T} 1$ significantly predicted longer attentional disengagement from negative to neutral information at high levels of adverse events, $B=0.10, S E=0.04, t=2.21, p=.03$. These effects are illustrated in Figure $4^{3}$. No other model (attentional disengagement from negative to positive, from positive to neutral, or from positive to negative) reached significance (see Table 4). ${ }^{4}$

\section{Further analyses: Control of potential confounders}

We ran further analyses to control for potential confounders in the relations supported between attentional disengagement and brooding in the main results. First, late-stage attentional

\footnotetext{
${ }^{3}$ Since the sample size for testing Moderation Model 2 was relatively small $(n=66)$, we further decomposed the brooding $\mathrm{x}$ adverse events interaction by also running conditional regressions using a more conservative medianbased splitting criterion. Conditional regressions with this more conservative approach again confirmed that higher brooding at $\mathrm{T} 1$ predicted faster attentional disengagement from negative to neutral information in the low adverse events level group, $B=-0.16, S E=0.07, t=-2.35, p=.03$. In contrast, and again confirming the main results with a splitting criteria based 1 SD above and below the mean of adverse events, higher brooding at T1 significantly predicted longer attentional disengagement from negative to neutral information in the high adverse events level group, $B=0.10, S E=0.03, t=2.77, p=.009$.

${ }^{4}$ All the prospective models considering the specific maladaptive component of brooding that are reported in the main manuscript were also performed using the global rumination score from the RRS instead. These further analyses showed that all statistically supported prospective moderation and moderated mediation models considering brooding levels were also significant when considering global rumination levels instead. Yet the strength of the effects was smaller in these models. This supports the higher relevance of specifically considering the brooding component on the interplays, rather than focusing on global rumination levels. These further analyses are available upon request.
} 
disengagement behaviors in the engagement-disengagement task were indexed following a $3,000 \mathrm{~ms}$ period of free viewing. Thus, this opens the possibility that individual differences in the main variables (i.e., time to move gaze away from one stimuli to engage with its counterpart) might be affected by individual differences in the time attending to the stimuli pair during the preceding free-viewing period. To discard this possibility, correlation analyses were performed between the proportion of time fixating toward emotional over neutral (or opposite emotional) facial expressions during the free-viewing period in each condition and their corresponding subsequent disengagement times. The Benjamini-Hochberg method was used to correct for false discovery rate for multiple comparisons. Analyses showed that preceding face processing during the free-viewing period did not correlate with the corresponding subsequent time to disengage from the target emotional face in neither of the four disengagement conditions (all $r$ 's $<.25$, all $p$ 's $>.05)$. We further wanted to replicate our moderated mediation model with disengagement from positive to negative at $\mathrm{T} 1$ as the predictor variable, while controlling for the preceding time attending to positive over negative faces during the free-viewing period in that condition as a covariate: step A was again supported, disengagement from positive to negative at T1 x adverse events interaction remained significant: $\Delta R^{2}=7.7 \%, F=7.58, p=.007$, while controlling for fixation time to positive over negative during the preceding free-viewing period, $B=-2.19, S E=$ $1.40, t=-1.56, p=.12$; step B modelling the association between depression change and brooding change was also confirmed, $B=0.23(S E=0.11), t=2.12, p=.04$, when partialling out the effects of the predictor (i.e., disengagement from positive to negative at T1), $B=-1.93(S E=1.91), t=-$ $1.01, p=.31$, as well as the effects of the fixation time to positive over negative during the preceding free-viewing period, $B=-2.18(S E=1.45), t=-1.50, p=.14$; finally, the moderated mediation index in step C remained statistically significant: $-0.83(\mathrm{SE}=.58), 95 \% \mathrm{CI}-2.4424--$ 0.0078. Further analyses were performed considering other attention indices collected during the 
free-viewing period (time to first fixate to the emotional faces, proportion of the number of fixations - fixation frequency - in emotional faces in the pair) that could also be potential confounders. In all cases, main findings remained unaffected by preceding free-viewing attention operations.

Second, we aimed to clarify whether differences in disengagement processes would be accounted by differential patterns of fixations when attending vs. when not attending to the target face-locations (the ones to be fixated in order to make the frame to appear at the opposite facelocation) at the end of the free viewing period. Overall, participants were fixating on the target face-location at the end of the free viewing period (i.e., beginning of the Wait-For-Fixation procedure) $40 \%$ of times $(\mathrm{SD}=.09)$ while they were not fixating on the target face-location (i.e., they were fixating on the opposite face or other location in between both stimuli) the remaining $60 \%$ of times $(\mathrm{SD}=.09)$. This distribution is logic and indicates that participants' attention was naturally distributed on the screen across trials at the end of the 3,000 ms free-viewing period. Further analyses compared disengagement times between each of those conditions confirming that disengagement times were not significantly different as a function of being attending or not to the target face-locations at the end of the free-viewing period (beginning of the Wait-ForFixation phase), $F(3,72)=1.13, p=.34, \eta p^{2}=.04$. To fully determine that potential inhibition of return effects were not affecting to subsequent disengagement patterns, we further modelled the association between participants' time to make the given fixation on the target face-location during the Wait-For-Fixation phase and their subsequent time to move gaze from that location to fixate on the opposite framed face-location during the Disengagement phase, on a trial basis and nested within-participants. The effect of time to fixate on the target face-location during the Wait- 
For-Fixation phase on the subsequent time to disengage from it during the Disengagement phase was almost negligible: $-0.04(S D=0.20), t=-0.21, p=.83$.

Finally, we aimed to replicate our moderated mediation for Hypothesis 1 with disengagement from positive to negative at $\mathrm{T} 1$ as the predictor variable, while controlling for general disengagement times in the neutral-neutral condition as a covariate. This contrast served to determine that observed relations in the main analyses were specific to the actual emotional predictor, not being confounded by systematic individual differences in other processes related to general disengagement time (i.e., general disengagement speed, peripheral shape stimulus registration sensitivity). All steps in the moderated mediation model were again replicated: step $\mathrm{A}, \Delta R^{2}=8 \%, F=7.71, p=.006$, while controlling for differences in general disengagement times in the neutral-neutral condition, $B=-2.12, S E=2.59, t=-0.83, p=.41$; step $\mathrm{B}, B=0.24(S E=$ $0.11), t=2.32, p=.02$, when partialling out the effects general disengagement times in the neutral-neutral condition, $B=-3.55(S E=2.52), t=-1.41, p=.16$; step $\mathrm{C}$, moderated mediation index $=-0.93(S E=.70), 95 \%$ CI $-3.0302--0.0038$.

\section{Discussion}

In the present study we tested the major predictions from the impaired disengagement framework (Koster et al., 2011), by relying on innovative techniques to track attentional disengagement processes (i.e., eye-tracking based measurements) at different emotional processing conditions. Our main results partially support the existence of the hypothesized bidirectional pathways of prospective influence between these late-stage attention disengagement biases and brooding.

First, longer times to disengage gaze from positive information were predictive of subsequent larger brooding decreases in individuals experiencing a higher frequency of adverse 
events. Moreover, this pathway also accounted for changes in depression levels, supporting the hypothesized moderated mediation model: longer disengagement from positive information at baseline indirectly predicted lower depression levels at follow-up (outcome), via their direct role on brooding (mediator) at high adverse event levels (moderator). Although very relevant, as we will discuss below, these results provide only partial support to the pathway hypothesized by Koster et al. (2011). Our results highlight a protective role of late-stage attention biases towards positive information (i.e., happy faces), whereas the first prediction of the impaired disengagement hypothesis indicates that the impaired attentional disengagement from negative information increases brooding in response to stress.

Second, an inverse pathway of influence between brooding and impaired disengagement from negative information (i.e., disgusted faces) was observed, supporting the second prediction of the impaired disengagement framework (i.e., habitual use of brooding reinforce subsequent impairments in attentional disengagement from negative information when coping with stress). Our results confirmed that individuals who report more habitual use of brooding and experienced high levels of adverse events show subsequent longer times to disengage gaze from negative information.

The differential pathways in our study may highlight differential roles of attention disengagement biases for positive and negative information on ruminative brooding not explicitly taken into account by the original impaired disengagement framework. The existence of both reduced maintenance on positive information and increased maintenance on negative information in habitual brooders has been shown in previous cross-sectional designs (e.g., Owens \& Gibbs, 2016). Yet, the specific contribution of each of these attention biases to mood (dys)regulation responses might occur at different stages. First, recent eye-tracking research shows that healthy individuals tend to increase attentional deployment towards positive stimuli in response to 
transient negative moods (Newman \& Sears, 2015; Sanchez, Vazquez, Gomez, \& Joormann, 2014) and that this specific pattern is predictive of subsequent efficient mood recovery (Sanchez et al., 2014). In line with this, longer times to disengage gaze from positive information in our unselected sample emerged as a protective factor, diminishing subsequent brooding responses when coping with adverse events and, in turn, indirectly predicting lower depression levels. Second, in the absence of such a protective positivity bias, brooding responses during the experience of adverse events might become more habitual across time. Once this occurs, it might subsequently impair disengagement from negative information when coping with new adverse events. Supporting this, and in line with recent empirical evidence (Valenas et al., 2017), the second prospective model in our study showed a predictive role of higher habitual use of brooding at baseline on subsequent longer attentional disengagement from negative information when coping with adverse events. Third, the emergence of impairments on negative disengagement as a result of stress might in turn reinforce subsequent brooding responses, as predicted by the impaired disengagement framework (Koster et al., 2011), in turn increasing depressive symptom levels. In the absence of additional time assessments in our longitudinal design, we cannot test this possibility. Yet, some indirect evidence suggests that specifically the co-occurrence of both negative attention biases and high habitual use of rumination in combination with high perceived stress may be the strongest predictor of subsequent higher depression levels (Morrison \& O’Connor, 2008) ${ }^{5}$.

\footnotetext{
${ }^{5}$ Morrison and Connor (2008) did not examine prospective relations between attention bias and rumination across time but, instead, how baseline negative attention bias (as measured with a dot-probe task) and high habitual ruminative responses interacted with the occurrence of perceived stress to prospectively predict depression. In that study, negative attention bias showed a trend towards interacting with rumination and stress to predict dysphoria three weeks later. We explored this further model in our data, with results supporting an identical three-way interaction trend $(B=-0.18, t=-1.93, p=.05)$ in the present longitudinal study. This further model in our study shows higher depression increases from $\mathrm{T} 1$ to $\mathrm{T} 2$ for those undergraduates coping with a higher degree of adverse events during the follow-up period who were characterized by both longer times to disengage gaze from negative information and higher brooding levels at the baseline assessment.
} 
Our main results highlight an attentional mechanism that may be protective against ruminative brooding, comprising maintained attention on positive information. Importantly, this predictor was identified using a positive vs. negative condition (i.e., disengaging from a positive face, when being prompted to engage with a negative counterpart), but not using a standard condition of disengagement (disengaging from a positive face, when being prompted to engage with a neutral counterpart). This suggests that the protective role of such disengagement bias might be specifically present in conditions comprising emotional conflict (e.g., maintaining attention on positive information when alternative information comprises negative rather than neutral information). Furthermore, our results also showed that this attention mechanism indirectly contributes to depression decreases via their role in brooding changes (step C, Model 1) but not directly (step B, Model 1). This is in line with previous cross-sectional research suggesting that the role of attention biases on depression may be indirect, via their influence on other intervening factors reflecting further elaborative mechanisms of mood (dys)regulation (interpretation, brooding, reappraisal: e.g, Everaert et al., 2016).

These results have relevant implications regarding the development of effective new strategies for depression prevention. Strategies aimed to reduce the risk to develop habitual and exaggerated brooding (and consequently depression) in response to stress may benefit from relying on techniques training top-down regulatory strategies to maximize maintained attention towards positive over negative features. New promising interventions, training top-down attention regulation to maximize maintained attention towards positive vs. competing negative information, have started to prove being effective to modify related mood regulation processes (Sanchez-Lopez, Koster, Van Put, Everaert, \& De Raedt, 2019; Sanchez, Everaert, \& Koster, 2016). In this new procedure, individuals are trained to allocate attention towards positive vs. negative words to efficiently create positive interpretations (i.e., implementing attentional 
control) while receiving contingent feedback on their gaze behavior. Sanchez et al. (2016) showed that higher attentional control implementation achieved during this training led to negative attention bias reduction, as measured with the dot-probe task, in turn predicting greater reappraisal success to reduce negative mood. Furthermore, in a recent study, Sanchez-Lopez et al. (2019) have used the engagement-disengagement attention task to measure transfer effects of this new training on changing gaze disengagement patterns, as the ones tested in the current study. Moreover, Sanchez-Lopez et al. (2019) replicated and extended initial findings showing that higher attentional control implementation achieved during this training resulted in longer times to disengage gaze from positive (happy) faces when prompted to engage with negative (disgusted) ones. This transfer, in turn, predicted greater reappraisal success to regulate negative mood when subsequently viewing negative scenes. Moreover, the extent to which trainees increased their attention towards positive over negative stimuli was also predictive of lower rumination while subsequently viewing negative scenes. Overall, online variants of this procedure might be a valuable tool to include as an add-on to currently ongoing rumination-focused cognitivebehavioral prevention protocols (e.g., Cook \& Watkins, 2016), since it may facilitate changes in attention mechanisms prospectively involved in the disruption of brooding responses to stress. Interestingly, whereas moderation analyses supported the second prediction that high occurrence of adverse events interacts with brooding to predict longer times to disengage gaze away from negative information, the opposite pattern was found at low occurrence of adverse events. Specifically, in the context of healthy functioning, our results suggest that, for individuals with low levels of brooding, the occurrence of adverse events is associated with faster disengagement of attention from negative information, while in the absence of stress, they invest longer times to disengage from this information. This intriguing result may suggest that healthy individuals (i.e., not characterized by high levels of brooding) are characterized by mechanisms 
of attentional flexibility (i.e., increasing or decreasing their attentional focus to negative features) as a function of temporally experienced stress, rather than by an overgeneralized faster disengagement from negative information. Further within-subject designs could shed light on this possibility and clarify whether this pattern may reflect mechanisms of individual's attention regulation or adjustment as a function of specifically encountered events, and whether contextbased attentional dynamic changes rather than temporally static attention biases may better facilitate healthier emotional functioning.

Despite the strength of our method and relevance of our findings, some limitations should be acknowledged. First, the use of only two time assessments in our longitudinal design limits our ability to test more complex interplays between temporal changes on attentional disengagement mechanisms, brooding, and depression in response to stress/adversity. Yet, our design comprised the evaluation of attention functioning using a rigorous eye-tracking procedure at two time assessments, being, to our knowledge, the first study to model differential pathways of prospective influence between direct indices of gaze disengagement and brooding. The longitudinal design employed was also rigorously applied to capture changes on the variables of interest right after a markedly adverse period (exams) for the population tested (undergraduates). However, this implies the evaluation of the variables of interest in response to mostly mild stressing events, which may not account for further casual interplays between impaired disengagement, brooding and depression in response to other more stressful or disabling conditions. Relatedly, the study was conducted with a largely homogenous non-clinical sample, limiting the generalizability of our results. For instance, it is plausible that this might have contributed to find more evidence in our study for protective effects (i.e., longer time to disengage from positive predicting brooding decreases) rather than for vulnerability effects (i.e., longer time to disengage from negative predicting brooding increases). Furthermore, although our 
sample was relatively large and attrition did not seem to have a substantial impact, future research will be necessary to replicate these findings using larger sample sizes. Finally, we assessed the occurrence of habitual brooding and adverse events during the same time frame. Future longitudinal studies would benefit from using more precise indices, such as assessing the specific occurrence of brooding responses to those adverse events (i.e., event-based brooding responses).

Another important issue refers to potential confounders that could affect the processes indexed in the engagement-disengagement task. As noted earlier, individual differences in the time to disengage attention might be affected by individual differences in the time attending to the stimuli pair during the preceding free-viewing period. However, we provide extensive evidence, further replicating our main findings while also controlling for potential confounders such as preceding attentional operations in the $3,000 \mathrm{~ms}$ period, differences in attentional location immediately before the beginning of the wait-for-fixation period, and control for other general individual differences in non-emotional disengagement behavior. Nonetheless, whilst these posthoc analyses substantively reduce the likelihood that these potential attentional confounds account for our results, they cannot definitively rule it out. In addition, individual differences in processes other than attention (e.g., stimulus encoding) could also operate to affect the measurement of attentional disengagement. Other researchers have argued that definitive assessment of attentional engagement and disengagement biases require that there is no prior processing of target stimuli prior to the measurement of selective attention so that logically there is no possibility of individual differences in pre-processing impacting on the assessment of engagement or disengagement indices (Clarke, MacLeod, \& Guastella, 2013). Further research is warranted to determine the extent to which these results are replicated with other versions of eyetracking tasks or when using versions of the dot-probe task modified to disentangle attentional engagement versus disengagement (Southworth et al., 2017). 
Despite these limitations, it is important to note that our study is the first to model prospective relations between attentional disengagement and brooding, using reliable and finegrained techniques of gaze disengagement assessment at several time points. We propose that future studies need to implement this sort of assessments into multi-wave longitudinal designs as well as into intensive ecological momentary assessments. Further research in this area would also benefit from extending the study of prospective mechanisms of impaired disengagement from negative information and brooding, as tested in the current study (i.e., disgusted faces), to other forms of negative information (i.e., angry and sad faces, lexical self-referent contents) with relevance to depression. These new advances will serve to clearly delineate dynamic pathways of interplay between attention mechanisms, emotion regulation processes, and depression, in order to develop the most efficient strategies for depression prevention. 


\section{Acknowledgements}

This research was supported by a grant of the Research Foundation Flanders (FWO) awarded to the first author and a grant BOF16/GOA/017 for Concerted Research Actions of Ghent University awarded to the second author and the last author. The first author is currently supported by the Program for the Attraction of Scientific Talent of the Community of Madrid. We are very grateful to Igor Marchetti for his assistance and comments on earlier versions of the manuscript. 


\section{References}

Aiken, L. S., \& West, S. G. (1991). Multiple regression: Testin and interpreting interactions. Multiple regression: Testing and interpreting interactions.

Aldao, A., Nolen-Hoeksema, S., \& Schweizer, S. (2010). Emotion-regulation strategies across psychopathology: A meta-analytic review. Clinical Psychology Review. https://doi.org/10.1016/j.cpr.2009.11.004

Amir, N., Klumpp, H., Elias, J., Bedwell, J. S., Yanasak, N., \& Miller, L. S. (2005). Increased activation of the anterior cingulate cortex during processing of disgust faces in individuals with social phobia. Biological Psychiatry, 57(9), 975-981. https://doi.org/10.1016/j.biopsych.2005.01.044

Armstrong, T., \& Olatunji, B. O. (2012). Eye tracking of attention in the affective disorders: A meta-analytic review and synthesis. Clinical Psychology Review, 32(8), 704-723. https://doi.org/10.1016/j.cpr.2012.09.004

Beck, A. T., Steer, R. A., \& Brown, G. K. (1996). Manual for the Beck Depression Inventory - II. San Antonio, TX: Psychological Corporation.

Beevers, C. G., \& Carver, C. S. (2003). Attentional bias and mood persistence as prospective predictors of dysphoria. Cognitive Therapy and Research, 27(6), 619-637. https://doi.org/10.1023/A:1026347610928

Beevers, C. G., Lee, H. J., Wells, T. T., Ellis, A. J., \& Telch, M. J. (2011). Association of predeployment gaze bias for emotion stimuli with later symptoms of PTSD and depression in soldiers deployed in Iraq. American Journal of Psychiatry, 168(7), 735-741. https://doi.org/10.1176/appi.ajp.2011.10091309

Benjamini, Y., \& Hochberg, Y. (1995). Controlling the false discovery rate: a practical and powerful approach to multiple testing. Journal of the Royal Statistical Society. Series B 
(Methodological), 57(1), 289-300. https://doi.org/10.2307/2346101

Blanco, I., Serrano-Pedraza, I., Vazquez, C. (2017). Don't look at my teeth when I smile: Teeth visibility in smiling faces affects emotionality ratings and gaze patterns. Emotion, 17(4), 640-647. https://doi.org/10.1037/emo0000260

Calvo, M. G., \& Lang, P. J. (2004). Gaze patterns when looking at emotional pictures: Motivationally biased attention. Motivation and Emotion, 28(3), 221-243. https://doi.org/10.1023/B:MOEM.0000040153.26156.ed

Carver, C. S. (1998). Generalization, adverse events, and development of depressive symptoms. Journal of Personality, 66(4), 607-619. https://doi.org/10.1111/1467-6494.00026

Chun, M. M., Golomb, J. D., \& Turk-Browne, N. B. (2010). A Taxonomy of External and Internal Attention. SSRN. https://doi.org/10.1146/annurev.psych.093008.100427

Clarke, P. J. F., MacLeod, C., \& Guastella, A. J. (2013). Assessing the role of spatial engagement and disengagement of attention in anxiety-linked attentional bias: a critique of current paradigms and suggestions for future research directions. Anxiety, Stress and Coping. https://doi.org/10.1080/10615806.2011.638054

Connolly, S. L., Wagner, C. A., Shapero, B. G., Pendergast, L. L., Abramson, L. Y., \& Alloy, L. B. (2014). Rumination prospectively predicts executive functioning impairments in adolescents. Journal of Behavior Therapy and Experimental Psychiatry, 45(1), 46-56. https://doi.org/10.1016/j.jbtep.2013.07.009

Cook, L., \& Watkins, E. (2016). Guided, internet-based, rumination-focused cognitive behavioural therapy (i-RFCBT) versus a no-intervention control to prevent depression in high-ruminating young adults, along with an adjunct assessment of the feasibility of unguided i-RFCBT, in the REdu. Trials, 17(1), 1. https://doi.org/10.1186/s13063-015-11289 
De Lissnyder, E., Koster, E. H. W., Goubert, L., Onraedt, T., Vanderhasselt, M. A., \& De Raedt, R. (2012). Cognitive control moderates the association between stress and rumination. Journal of Behavior Therapy and Experimental Psychiatry, 43(1), 519-525. https://doi.org/10.1016/j.jbtep.2011.07.004

Demeyer, I., De Lissnyder, E., Koster, E. H. W., \& De Raedt, R. (2012). Rumination mediates the relationship between impaired cognitive control for emotional information and depressive symptoms: A prospective study in remitted depressed adults. Behaviour Research and Therapy, 50(5), 292-297. https://doi.org/10.1016/j.brat.2012.02.012

Donaldson, C., Lam, D., \& Mathews, A. (2007). Rumination and attention in major depression. Behaviour Research and Therapy, 45(11), 2664-2678. https://doi.org/10.1016/j.brat.2007.07.002

Duque, A., Sanchez, A., \& Vazquez, C. (2014). Gaze-fixation and pupil dilation in the processing of emotional faces: The role of rumination. Cognition and Emotion, 28(8), 1347-1366. https://doi.org/10.1080/02699931.2014.881327

Everaert, J., Grahek, I., Duyck, W., Buelens, J., Van den Bergh, N., \& Koster, E. H. W. (2016). Mapping the interplay among cognitive biases, emotion regulation, and depressive symptoms. Cognition and Emotion, (August). https://doi.org/10.1080/02699931.2016.1144561

Hayes, A. F. (2012). PROCESS: A versatile computational tool for observed variable mediation, moderation, and conditional process modeling. White Paper. https://doi.org/978-1-60918$230-4$

Joormann, J., Dkane, M., \& Gotlib, I. H. (2006). Adaptive and Maladaptive Components of Rumination? Diagnostic Specificity and Relation to Depressive Biases. Behavior Therapy, 37(3), 269-280. https://doi.org/10.1016/j.beth.2006.01.002 
Joormann, J., \& Quinn, M. E. (2014). Cognitive processes and emotion regulation in depression. In Depression and Anxiety (Vol. 31, pp. 308-315). https://doi.org/10.1002/da.22264

Kashdan, T. B., \& Roberts, J. E. (2007). Social anxiety, depressive symptoms, and post-event rumination: Affective consequences and social contextual influences. Journal of Anxiety Disorders, 21(3), 284-301. https://doi.org/10.1016/j.janxdis.2006.05.009

Koster, E. H. W., De Lissnyder, E., Derakshan, N., \& De Raedt, R. (2011). Understanding depressive rumination from a cognitive science perspective: The impaired disengagement hypothesis. Clinical Psychology Review, 31(1), 138-145.

https://doi.org/10.1016/j.cpr.2010.08.005

Langner, O., Dotsch, R., Bijlstra, G., Wigboldus, D. H. J., Hawk, S. T., \& van Knippenberg, A. (2010). Presentation and validation of the Radboud Faces Database. Cognition \& Emotion, 24(8), 1377-1388. https://doi.org/10.1080/02699930903485076

Lyubomirsky, S., \& Tkach, C. (2008). The Consequences of Dysphoric Rumination. In

Depressive Rumination: Nature, Theory and Treatment (pp. 21-41). https://doi.org/10.1002/9780470713853.ch2

Morrison, R., \& O'Connor, R. C. (2008). The role of rumination, attentional biases and stress in psychological distress. British Journal of Psychology (London, England: 1953), 99(Pt 2), 191-209. https://doi.org/10.1348/000712607X216080

Muller, D., Judd, C. M., \& Yzerbyt, V. Y. (2005). When moderation is mediated and mediation is moderated. Journal of Personality and Social Psychology. https://doi.org/10.1037/00223514.89.6.852

Newman, K. R., \& Sears, C. R. (2015). Eye Gaze Tracking Reveals Different Effects of a Sad Mood Induction on the Attention of Previously Depressed and Never Depressed Women. Cognitive Therapy and Research, 39(3), 292-306. https://doi.org/10.1007/s10608-014-9669- 
$\mathrm{X}$

Nolen-Hoeksema, S. (1991). Responses to depression and their effects on the duration of depressive episodes. Journal of Abnormal Psychology, 100(4), 569-582. https://doi.org/10.1037/0021-843X.100.4.569

Nolen-Hoeksema, S. (2000). The role of rumination in depressive disorders and mixed anxiety/depressive symptoms. Journal of Abnormal Psychology, 109(3), 504-511. https://doi.org/10.1037/0021-843X.109.3.504

Nolen-Hoeksema, S., McBride, a, \& Larson, J. (1997). Rumination and psychological distress among bereaved partners. Journal of Personality and Social Psychology, 72(4), 855-862. https://doi.org/10.1037/0022-3514.72.4.855

Nolen-Hoeksema, S., \& Morrow, J. (1991). A Prospective Study of Depression and Posttraumatic Stress Symptoms After a Natural Disaster: The 1989 Loma Prieta Earthquake. Journal of Personality and Social Psychology, 61(1), 115-121. https://doi.org/10.1037/00223514.61.1.115

Nolen-Hoeksema, S., Parker, L. E., \& Larson, J. (1994). Ruminative coping with depressed mood following loss. Journal of Personality and Social Psychology, 67(1), 92-104. https://doi.org/10.1037/0022-3514.67.1.92

Owens, M., \& Gibb, B. E. (2017). Brooding rumination and attentional biases in currently nondepressed individuals: an eye-tracking study. Cognition and Emotion, 31(5), 1062-1069. https://doi.org/10.1080/02699931.2016.1187116

Raes, F., \& Hermans, D. (2007). The revised version of the Dutch Ruminative Response Scale. Unpublished Instrument. 
Sanchez-Lopez, A., Koster, E. H. W., Van Put, J., Everaert, J., \& De Raedt, R. (2019). Eye-gaze contingent attention training (ECAT):Examining the causal role of attention regulation in reappraisal and rumination. Biological Psychology.

https://doi.org/10.1016/j.biopsycho.2019.01.017

Sanchez, A., Everaert, J., \& Koster, E. H. W. (2016). Attention training through gaze-contingent feedback: Effects on reappraisal and negative emotions. Emotion, 16(7). https://doi.org/10.1037/emo0000198

Sanchez, A., Romero, N., \& De Raedt, R. (2017). Depression-related difficulties disengaging from negative faces are associated with sustained attention to negative feedback during social evaluation and predict stress recovery. PLoS ONE, 12(3), 1-24. https://doi.org/10.1371/journal.pone.0175040

Sanchez, A., Romero, N., \& De Raedt, R. (2017). Depression-related difficulties disengaging from negative faces are associated with sustained attention to negative feedback during social evaluation and predict stress recovery. PLoS ONE, 12(3). https://doi.org/10.1371/journal.pone.0175040

Sanchez, A., Vanderhasselt, M.-A., Baeken, C., \& De Raedt, R. (2016). Effects of tDCS over the right DLPFC on attentional disengagement from positive and negative faces: An eyetracking study. Cognitive, Affective, \& Behavioral Neuroscience, 16(6), 1027-1038. https://doi.org/10.3758/s13415-016-0450-3

Sanchez, A., Vazquez, C., Gomez, D., \& Joormann, J. (2014). Gaze-fixation to happy faces predicts mood repair after a negative mood induction. Emotion (Washington, D.C.), 14(1), 85-94. https://doi.org/10.1037/a0034500 
Sanchez, A., Vazquez, C., Marker, C., LeMoult, J., \& Joormann, J. (2013). Attentional disengagement predicts stress recovery in depression: an eye-tracking study. Journal of Abnormal Psychology, 122(2), 303-313. https://doi.org/10.1037/a0031529

Southworth, F., Grafton, B., MacLeod, C., \& Watkins, E. (2017). Heightened ruminative disposition is associated with impaired attentional disengagement from negative relative to positive information: support for the "impaired disengagement" hypothesis. Cognition and Emotion, 31(3), 422-434. https://doi.org/10.1080/02699931.2015.1124843

Szent, A., Miu, A. C., \& Macleod, C. (2017). Behaviour Research and Therapy Prediction of preexam state anxiety from ruminative disposition: The mediating role of impaired attentional disengagement from negative information lenas, 91, 102-110.

https://doi.org/10.1016/j.brat.2017.01.014

Treynor, W., Gonzalez, R., \& Nolen-Hoeksema, S. (2003). Rumination reconsidered: A psychometric analysis. Cognitive Therapy and Research, 27(3), 247-259. https://doi.org/10.1023/A:1023910315561

Van der Does, A. J. W. (2002). Handleiding bij de Nederlandse versie van de Beck Depression Inventory - second edition (BDI-II-NL). [The Dutch version of the Beck Depresion Inventory-II]. Lisse, The Netherlands: Swets \& Zeitlinger.

Vanderhasselt, M. A., Brose, A., Koster, E. H. W., \& De Raedt, R. (2016). Co-variation between stressful events and rumination predicts depressive symptoms: An eighteen months prospective design in undergraduates. Behaviour Research and Therapy, 87, 128-133. https://doi.org/10.1016/j.brat.2016.09.003

Waechter, S., Nelson, A. L., Wright, C., Hyatt, A., \& Oakman, J. (2014). Measuring attentional bias to threat: Reliability of dot probe and eye movement indices. Cognitive Therapy and Research, 38(3). https://doi.org/10.1007/s10608-013-9588-2 
Watkins, E. R. (2008). Constructive and unconstructive repetitive thought. Psychological Bulletin, 134(2), 163-206. https://doi.org/10.1037/0033-2909.134.2.163

Yzerbyt, V., Muller, D., Batailler, C., \& Judd, C. M. (2018). New recommendations for testing indirect effects in mediational models: The need to report and test component paths. Journal of Personality and Social Psychology, 115(6), 929-943. https://doi.org/10.1037/pspa0000132

Zetsche, U., \& Joormann, J. (2011). Components of interference control predict depressive symptoms and rumination cross-sectionally and at six months follow-up. Journal of Behavior Therapy and Experimental Psychiatry, 42(1), 65-73. https://doi.org/10.1016/j.jbtep.2010.06.001 
Table 1. Descriptives and internal consistencies of variables in the study at each assessment time.

\begin{tabular}{lcccccc}
\hline & \multicolumn{2}{c}{ Time 1} & & \multicolumn{2}{c}{ Time 2} \\
\cline { 2 - 3 } \cline { 5 - 6 } Variables & M (SD) & $\alpha$ & & M (SD) & $\alpha$ \\
\hline BDI-II - Depressive Symptoms & $6.31(5.71)$ & .85 & & $6.39(5.74)$ & .86 \\
RRS - Brooding & $10.98(3.27)$ & .71 & & $10.44(3.56)$ & .80 \\
Att. Diseng. from Neg to Neu (ms) & $268(58)$ & .71 & & $276(58)$ & .63 \\
Att. Diseng. from Neg to Pos (ms) & $263(57)$ & .63 & $270(63)$ & .60 \\
Att. Diseng. from Pos to Neu (ms) & $266(58)$ & .67 & & $265(52)$ & .65 \\
Att. Diseng. from Pos to Neg (ms) & $274(57)$ & .64 & $282(65)$ & .71 \\
\hline
\end{tabular}

Notes. $M=$ Mean; $S D=$ Standard deviation; $\alpha=$ alpha (internal consistency) BDI-II = Beck Depression Inventory II; RRS = Ruminative Responses Scale

Att. Diseng= Late-stage attentional disengagement; Neg= Negative faces (disgusted); Pos= Positive faces (happy); Neu $=$ Neutral faces $; \mathrm{ms}=$ milliseconds 
Table 2. Correlations between variables of interest at each assessment time

\begin{tabular}{|c|c|c|c|c|c|c|c|c|c|c|c|c|}
\hline \multirow[t]{2}{*}{ Variables } & \multicolumn{6}{|c|}{ Time 1} & \multicolumn{6}{|c|}{ Time 2} \\
\hline & (1) & (2) & (3) & (4) & (5) & (6) & (1) & (2) & (3) & (4) & $(5)$ & (6) \\
\hline (1) BDI-II - Depressive Symptoms & - & & & & & & - & & & & & \\
\hline (2) RRS - Brooding & $.38 * *$ & - & & & & & $.25^{*}$ & - & & & & \\
\hline (3) Att. Diseng. from Neg to Neu (ms) & .05 & .12 & - & & & & .02 & .02 & - & & & \\
\hline (4) Att. Diseng. from Neg to Pos (ms) & .08 & .03 & $.65^{* *}$ & - & & & .12 & .01 & $.45^{* *}$ & - & & \\
\hline (5) Att. Diseng. from Pos to Neu (ms) & .06 & .10 & $.58 * *$ & $.58 * *$ & - & & .09 & .11 & $.34 * *$ & $.65^{* *}$ & - & \\
\hline (6) Att. Diseng. from Pos to Neg (ms) & .06 & .03 & $.63 * *$ & $.44 * *$ & $.50 * *$ & - & .03 & .04 & $.53 * *$ & $.62 * *$ & $.59 * *$ & - \\
\hline
\end{tabular}

Notes. $* p<.05 ; * * p<.01$

$B$ DI-II = Beck Depression Inventory II; RRS = Ruminative Responses Scale

Att. Diseng= Late-stage attentional disengagement; Neg= Negative faces (disgusted); Pos= Positive faces (happy); Neu= Neutral faces; ms= milliseconds 
Table 3. Predictive role of attention disengagement indices at $\mathrm{T} 1$ in brooding changes as a function of adverse events

\begin{tabular}{|c|c|c|c|c|c|c|c|}
\hline \multirow[b]{2}{*}{ Predictor T1 } & \multicolumn{4}{|c|}{ Direct effect on Brooding Change } & \multicolumn{3}{|c|}{ Interaction with Adverse Events on Brooding Change } \\
\hline & $B$ & $S E$ & $t$ & $p$ & $\Delta R 2$ & $\Delta F$ & $p$ \\
\hline $\begin{array}{l}\text { Attentional disengagement } \\
\text { from negative to neutral }\end{array}$ & 1.33 & 2.54 & 0.52 & 0.60 & $2.3 \%$ & 2.11 & 0.15 \\
\hline $\begin{array}{l}\text { Attentional disengagement } \\
\text { from negative to positive }\end{array}$ & 2.24 & 2.53 & 0.89 & 0.38 & $3.2 \%$ & 2.92 & 0.15 \\
\hline $\begin{array}{l}\text { Attentional disengagement } \\
\text { from positive to neutral }\end{array}$ & 2.60 & 2.19 & 1.18 & 0.24 & $6.1 \%$ & 5.61 & 0.02 \\
\hline $\begin{array}{l}\text { Attentional disengagement } \\
\text { from positive to negative }\end{array}$ & 2.08 & 2.43 & 0.86 & 0.39 & $7.4 \%$ & 7.08 & 0.02 \\
\hline
\end{tabular}

Notes. In bold: models where significant interaction effects were supported. $P$ values corrected controlling for false discovery rate in multiple testing, using the Benjamini-Hochberg procedure. 
Table 4. Predictive role of brooding at T1 in attention disengagement changes as a function of adverse events

\begin{tabular}{|c|c|c|c|c|c|c|c|}
\hline \multirow[b]{2}{*}{ Outcome Change in: } & \multicolumn{4}{|c|}{ Direct effect of Brooding at T1 } & \multicolumn{3}{|c|}{ Adverse Events by Brooding T1 Interaction } \\
\hline & $B$ & $S E$ & $t$ & $p$ & $\Delta R 2$ & $\Delta F$ & $p$ \\
\hline $\begin{array}{l}\text { Attentional disengagement } \\
\text { from negative to neutral }\end{array}$ & -0.14 & 0.05 & -2.75 & 0.02 & $16.7 \%$ & 12.87 & 0.002 \\
\hline $\begin{array}{l}\text { Attentional disengagement } \\
\text { from negative to positive }\end{array}$ & $B=-0.05$ & 0.05 & -0.86 & 0.39 & $1.3 \%$ & 0.91 & 0.35 \\
\hline $\begin{array}{l}\text { Attentional disengagement } \\
\text { from positive to neutral }\end{array}$ & $\mathrm{B}=-0.03$ & 0.05 & -0.54 & 0.59 & $0.1 \%$ & 0.12 & 0.73 \\
\hline $\begin{array}{l}\text { Attentional disengagement } \\
\text { from positive to negative }\end{array}$ & $B=-0.06$ & 0.05 & -1.06 & 0.58 & $0.2 \%$ & 0.76 & 0.68 \\
\hline
\end{tabular}

Notes. In bold: models where significant main effects of the predictor and interaction effects with the moderator were supported. $P$ values corrected controlling for false discovery rate in multiple testing, using the Benjamini-Hochberg procedure. 
Figure 1. Models tested in the longitudinal study

Fig 1a. Moderated Mediation, Hypothesis 1

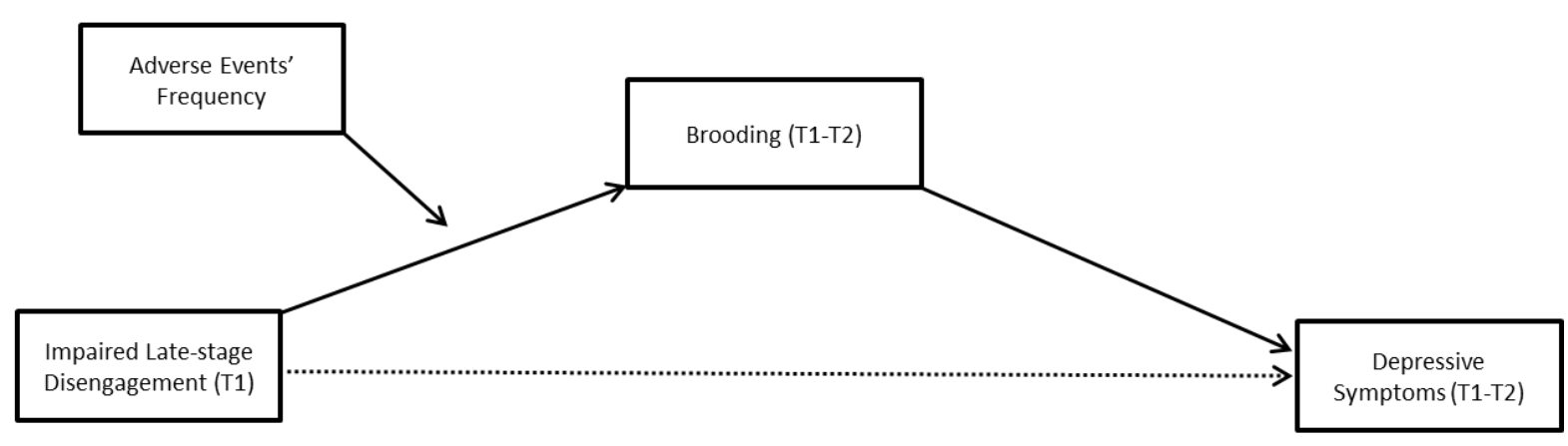

Fig 1b. Moderation, Hypothesis 2

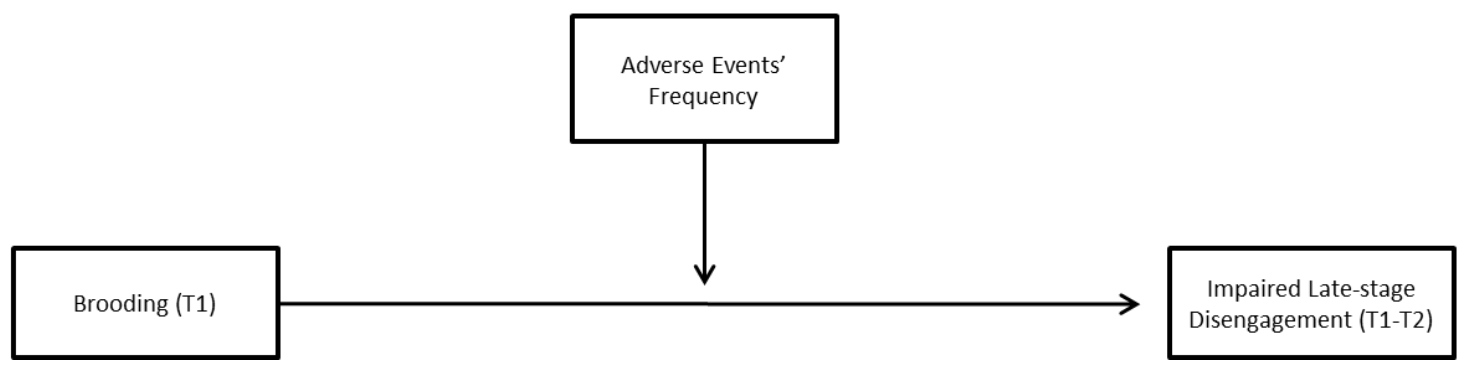


Figure 2. Trials' sequences and measures assessed for each attentional disengagement index
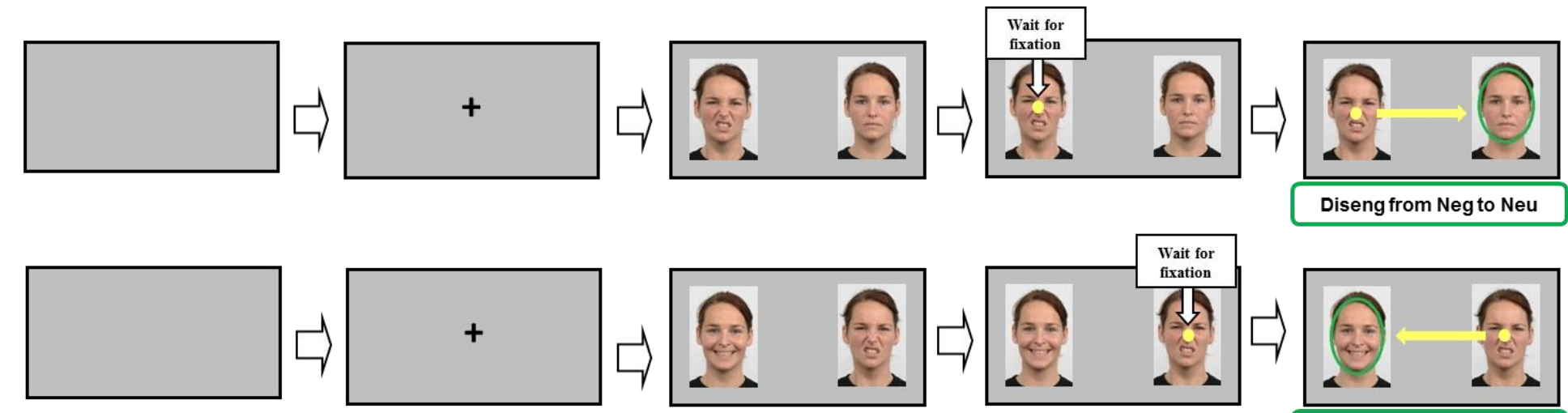

Diseng from Neg to Neu
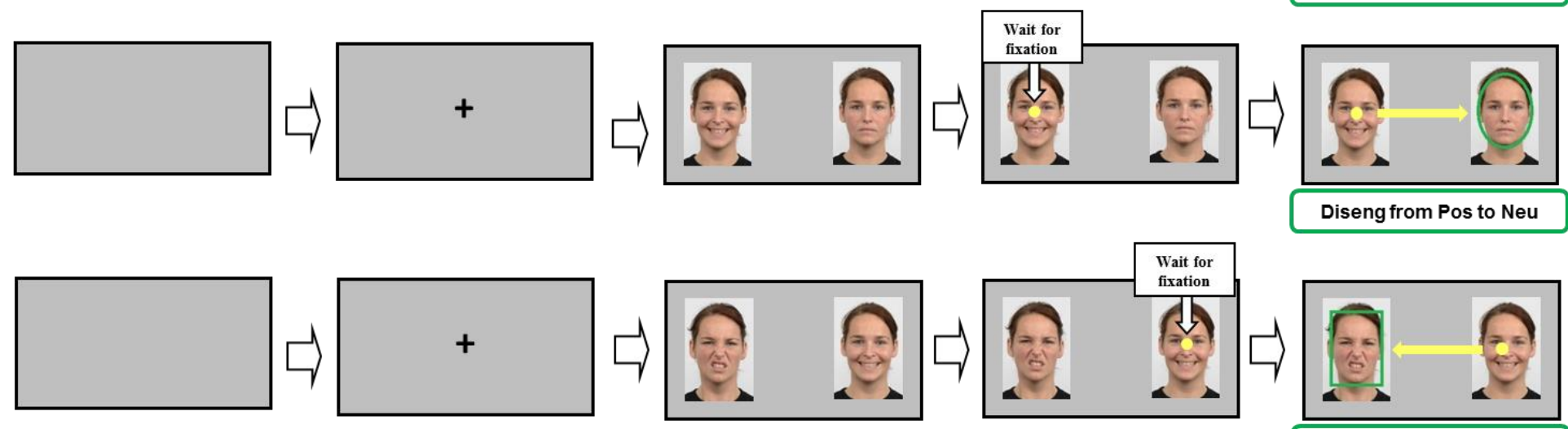

Diseng from Pos to Neu
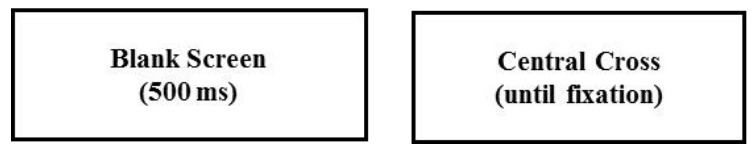

$$
\begin{aligned}
& \text { Free viewing of face } \\
& \text { pairs }(3000 \mathrm{~ms})
\end{aligned}
$$
Wait for Fixation $100 \mathrm{~ms}$ fixating on the target face)

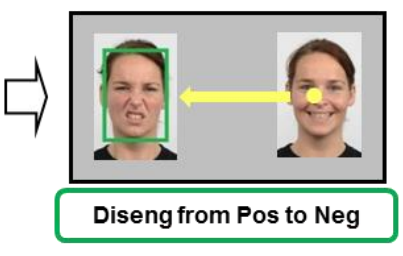

Notes. Neg= Negative face $($ disgusted); Pos= Positive face (happy); Neu= Neutral face 
Figure 3. Moderated effects as a function of adverse events' frequency in step A, Hypothesis 1

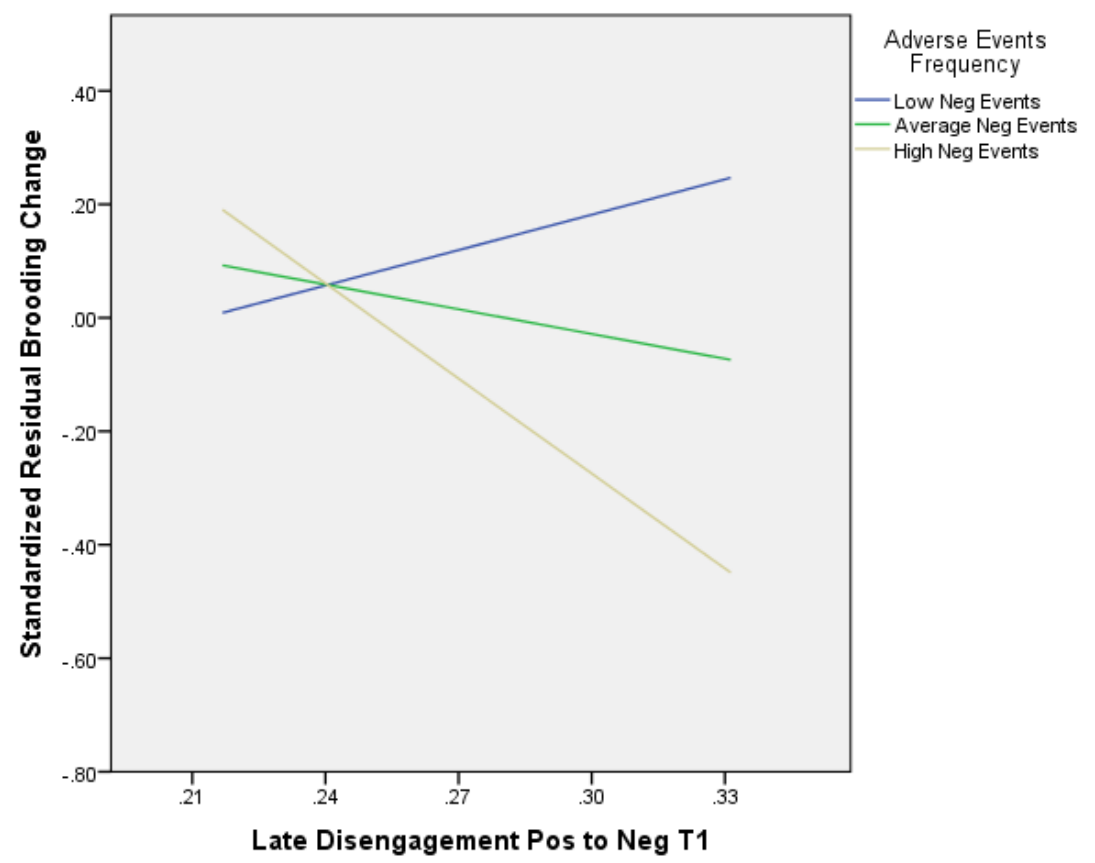

Notes. T1= Assessment Time 1; Neg Events= Negative adverse events during follow-up period; Neg= Negative faces (disgusted); Pos= Positive faces (happy) 
Figure 4. Moderated effects as a function of adverse events' frequency in step A, Hypothesis 2

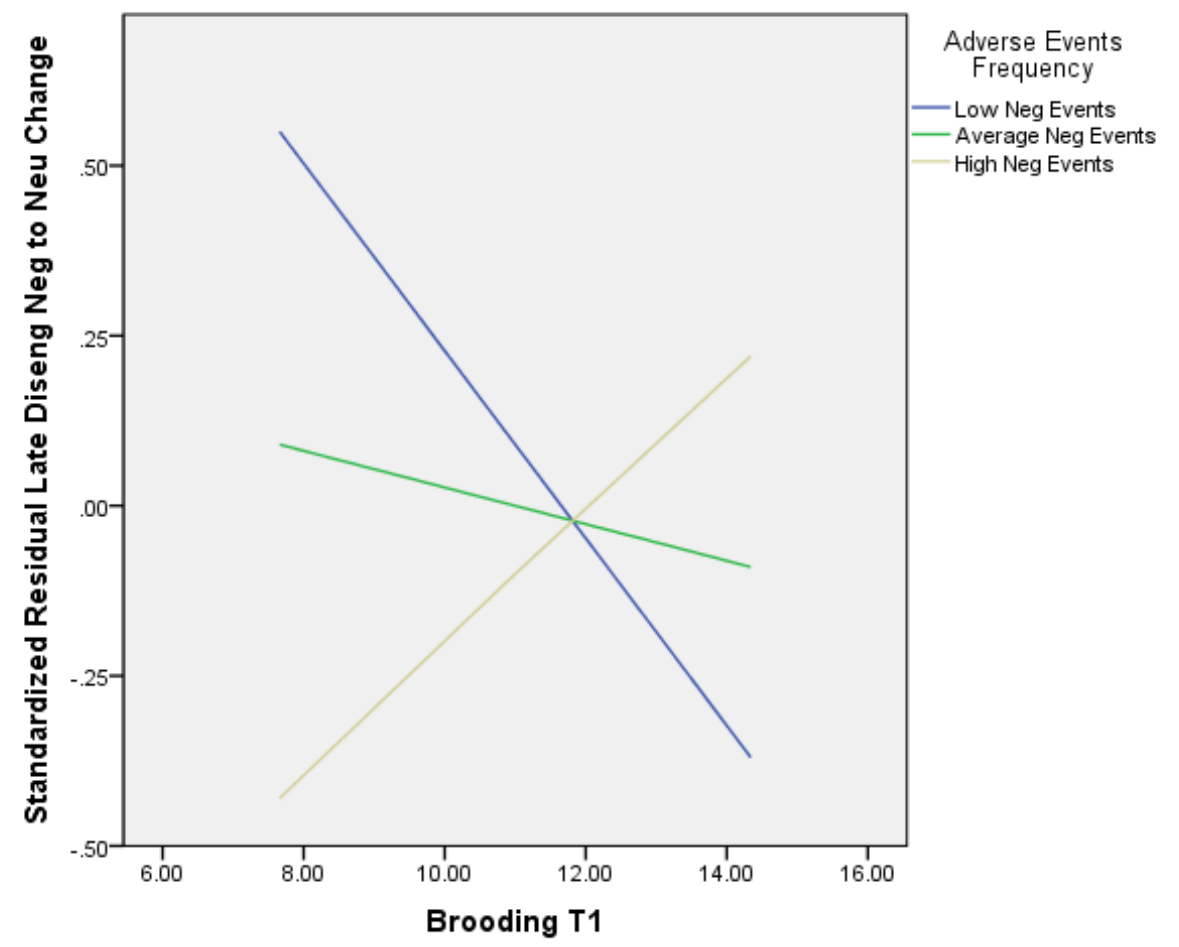

Notes. $\mathrm{T} 1=$ Assessment Time 1; Neg Events= Negative adverse events during follow-up period; Neg= Negative faces (disgusted); Neu= Neutral faces 